\title{
Stability and Retraction Force Verification of a New Retractor Design for Minimally Invasive Surgery
}

\author{
Illés Nigicser \\ Department of Mechanical Engineering \\ Sciences, Faculty of Engineering and \\ Physical Sciences \\ University of Surrey \\ Guildford, UK \\ nigicseri@gmail.com
}

\author{
Matthew Oldfield \\ Department of Mechanical Engineering \\ Sciences, Faculty of Engineering and \\ Physical Sciences \\ University of Surrey \\ Guildford, UK \\ m.oldfield@surrey.ac.uk
}

\author{
Tamás Haidegger \\ University Research and Innovation Center \\ (EKIK) / John von Neumann Faculty of \\ Informatics (NIK) \\ Óbuda University, Budapest, Hungary \\ haidegger@irob.uni-obuda.hu
}

\begin{abstract}
Minimally Invasive Surgery (MIS) needs continuous tool design innovation to support and facilitate the complex task executions of surgeons. In this article, an easily deployable magnetic structure design is presented, which is developed to retract the liver during MIS procedures. During the concept designing phase, a most critical research question, the stability of magnetic anchoring was investigated and analyzed through various experiments. The clinically relevant pulling forces have been applied to $\mathrm{N} 52$ neodymium magnets in different size, shape and arrangement to derive the maximum force certain retractor designs could withheld. The numeric results confirmed that the distributed load arrangement would be able to perform a stable human liver retraction. Magnetic encoring technology could have a significant future, encouraging other researchers to investigate the potential of magnetic tissue retraction in MIS procedures that could lead to the development of specialized tools for human clinical deployment.
\end{abstract}

Keywords-Minimally Invasive Surgery tool, magnetic anchoring, liver retraction, folding surgical tool design

\section{INTRODUCTION}

Minimally Invasive Surgery (MIS) procedures, whether done manually with laparoscopic instruments or robotically, present significant clinical benefits, yet conversely some technical challenges, when compared to traditional open surgery [1-3]. Currently, the development of autonomous robotic solutions is in the focus of many research initiatives [4], while realistically, refined manual tool can already significantly ease the complexity of the MIS procedures, and therefore potentially improve the clinical outcome. In MIS, establishing the necessary working volume for various procedures is a challenge due to the limited space and access. In order to ensure sufficient working volume and visual clarity, while limiting the invasiveness, tissues and organs surrounding the procedure target must be retracted or lifted away. Therefore, tissue retraction is critically important. Based on the current literature and surgical device portfolios it can be stated that techniques and solutions for retraction in MIS procedures lack efficiency and practicality.

There are a number of lower and upper abdominal procedures that need tissue retraction in various forms and degree. Such lower abdominal procedures include hysterectomy, prostatectomy, colectomy and hernia repairs. The bowels can be retracted by using an additional incision, where an assisting surgeon uses an additional mechanical laparoscopic instrument to retract the bowel at certain parts of the procedure. MIS retractors are often unfoldable, like a fan or an umbrella. The efficiency of the retraction can be improved by optimizing the instrument used for retraction, an example of which is an inflatable atraumatic retractor or magnetic anchoring, however, these pose numerous engineering design and application challenges with respect to safe insertion, deployment and retraction [5].

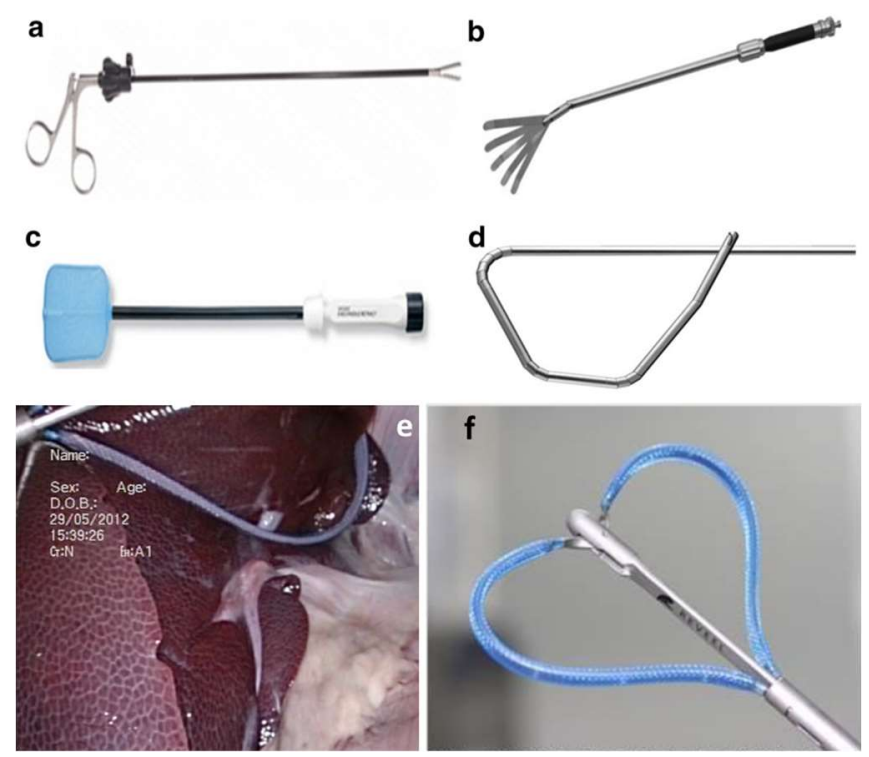

Figure 1 - Retractors used in MIS: a) Laparoscopic bowel graspers (Medline Industries). b) Laparoscopic Fan retractor (LocaMed Ltd). c) Inflatable Endo Paddle retractor (Covidien Surgical). d) Snowden-Pencer triangular liver retractor (Cardinal Health). e) Kelly Liver Retractor in deployment (Surtex Instruments). f) Inflatable design from the Chinese University Hong Kong [5, 8]. 
The liver is a delicate tissue [6], often in the way of common MIS procedures, such as cholecystectomy [7]. A less invasive and more efficient liver retraction solution would reduce tissue damage, improve patient outcome and therefore significantly reduce hospital stay and consequently hospital costs.

Moreover, it would greatly impact the work of the surgeons and surgical staff providing them with liver retraction solution that is easier to apply, reduces the technical challenge and lessens intra-operative mental stress. This would allow the surgeons to focus on the main surgical tasks with fewer distractions. Many clinicians are reluctant to use traditional liver retractors altogether because of their impracticality. With new MIS tool design solutions, however, they would be encouraged to sufficiently retract the liver without compromising the quality of the procedure.

\section{METHODS}

\section{A. Basic functional requirements}

The engineering design was initiated with identifying the clinical and user requirements. Many tissues are handled via retractors during MIS. Among the large abdominal organs, such as the liver, stomach and bowels, the liver was chosen since it covers and obstructs the largest workspace of abdominal procedures, and the retraction of which still causes significant challenges due to its large size. Based on a 2018 study it was determined that $11.25 \mathrm{~N}$ force is required to retract the liver [9]. The liver retraction solution therefore will focus on achieving sufficient lifting of the right or the left lobe of the liver where the former is necessary for cholecystectomy procedures where the Calot's triangle needs clear exposure [10] and the latter for bariatric procedures where the hiatus needs exposure [11]. Stability is guaranteed through these forces already, since in MIS, typically a $10 \mathrm{~N}$ force limit is expected on the tools during the operations, thus the structure could withstand those.

Clinical/medical device requirements, such as human surgery grade material choice, sterilization and manufacturability have not been considered in this early phase of the research

\section{B. Design considerations}

The fundamental idea of the magnetic solution was to design an insertable magnetic structure that would act as inter-

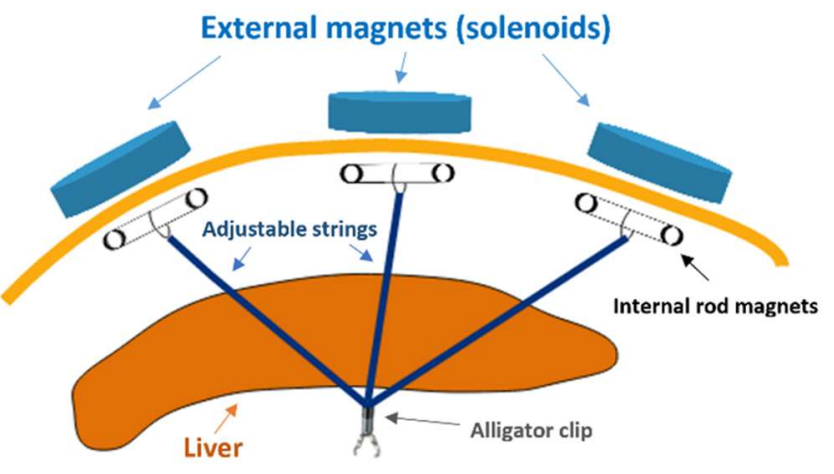

Figure 1 - Initial design concept of a magnetic anchoring system with external magnets, internal rod magnets and adjustable strings, facilitating sideways folding of the liver.

\section{a) Compact folded state}

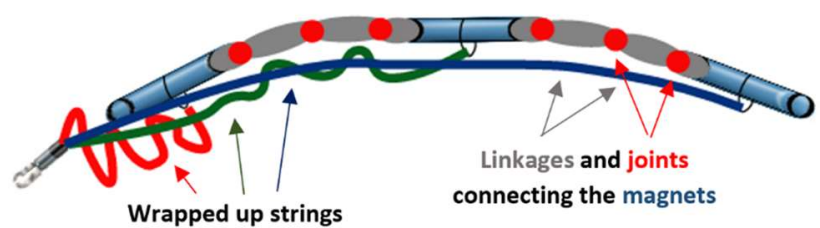

b) Deployed state

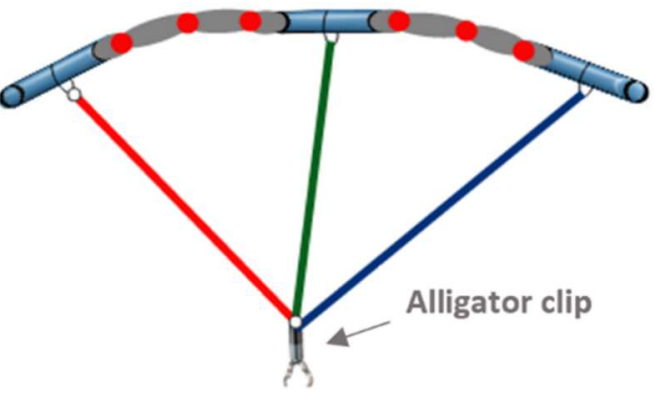

Figure 2 - Deployable anchoring magnetic structure with adjustable strings in a) folded state to fit through a $10 \mathrm{~mm}$ diameter trocar wrapped up and b) in a deployed state inside the abdomen.

nal anchor points for liver retraction, so that the abdominal wall could remain intact during retraction. In this concept, the distance between the insertable magnetic structure and the external magnet would be equivalent to the abdominal wall thickness, over which sufficient magnetic force would need to be maintained so that the internal structure could support the weight of the liver [9]. This solution would consist of multiple internal rod magnets, each anchored externally by magnets positioned outside the human body. The internal rod magnets could be used as internal anchor points for adjustable strings that would retract the liver by hooping underneath it or by using an alligator clip to grasp the right crus of the diaphragm underneath the liver to facilitate sideways folding of the liver. This initial solution concept is detailed in Fig. 1.

\section{Optimized magnetic anchoring}

In order to prevent the internal magnets to collide with each other (and cause difficulty to be separated from each other), these rod magnets could be connected to each other by joints and linkages that limit their range of motion. This would result in a single deployable structure that is to be inserted and removed through a $10 \mathrm{~mm}$ diameter trocar port. Such a design concept is illustrated in Fig. 2.

\section{MEGNETIC EXPERIMENTS FOR ATTACHMENT}

The experimental work aimed to simulate magnetic forces as a function of distance, the use of various magnetic and ferromagnetic target specimens and the effect of the environmental medium. The results were expected to give a critically evaluated foundation to the detailed design of the magnetic retractor system.

The experiments were performed on an Instron electromechanical universal testing machine, capable of performing a variety of tensile and compressive tests. More 
specifically, the Dual Column Tabletop Testing System (Fig. 3), was capable of measuring up to $50 \mathrm{kN}$ force capacity. This experimental machine allowed for testing magnetic force as a function of distance with high accuracy. It was achieved by attaching the permanent magnet to the lower leg and the target specimen to the upper leg of the instrument. The distance between the upper and lower leg could be adjusted, measured and varied through the user interface. The upper leg contained the load cell with $500 \mathrm{~Hz}$ data acquisition rate.

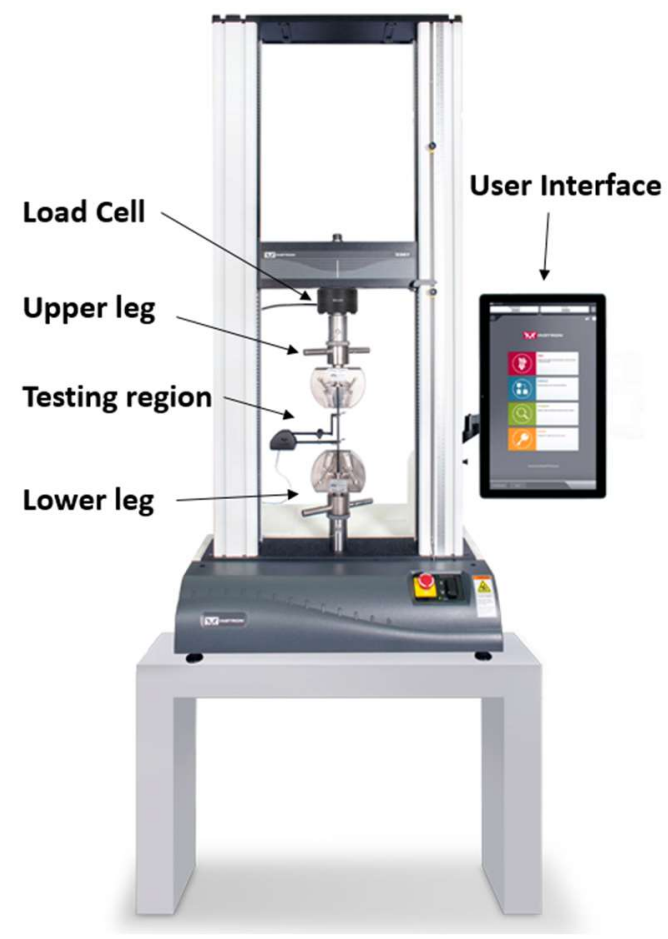

Figure 1 - Instron Dual Column Tabletop Testing System image courtesy of Instron (Instron, 2019).

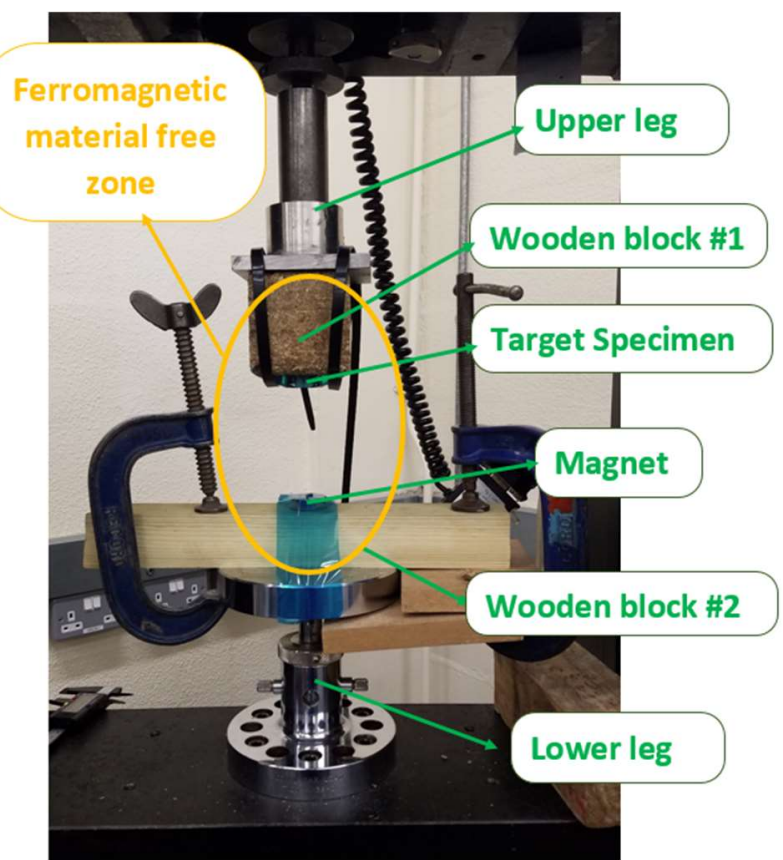

Figure 4-Experimental setup of testing the magnetic force as a function of distance on the Instron machine with a mild steel and a silver steel target specimen against a $24 \mathrm{~kg}$ pull force N52 neodymium disk magnet.

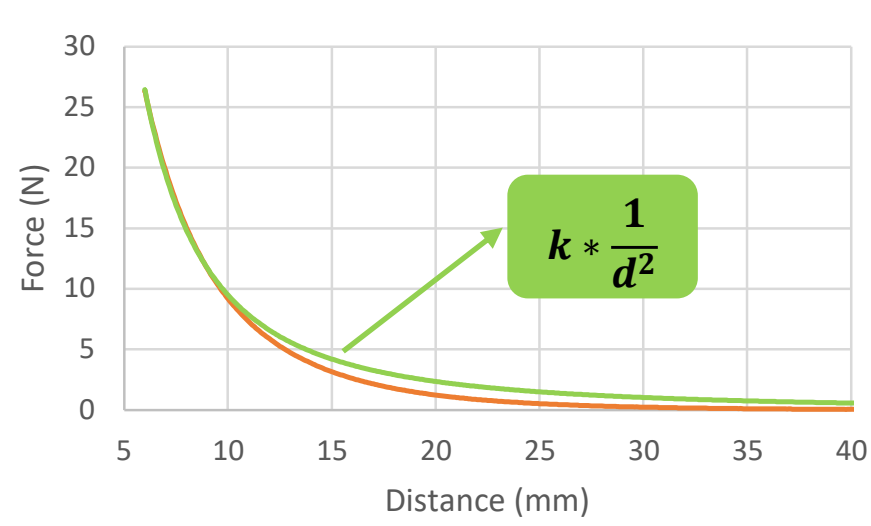

silver steel _ inverse square function

Figure 5 - Comparative results of the experimental force curve and the expected scaled inverse square law, where $d$ represents the distance between the magnet and the target.

Each of the experiments performed provided notable findings about the magnetic technology and directions for the use of this technology in this research. Experiment 1 established the behavior of the magnetic force as a function of increased distance (Fig. 4) and confirmed the theoretical inverse square law when a cylindrical steel disk is distanced from a cylindrical permanent disk magnet of the same dimensions. A grade N52 neodymium cylindrical disk magnet was used that was magnetised along its thickness. It had a diameter of $30 \mathrm{~mm}$, a thickness of $10 \mathrm{~mm}$ and a commercial rating of $24 \mathrm{~kg}$ pull-force. Two different target specimens were used of the same dimensions as the magnet, one made of mild steel and the other of silver steel. Both measurements followed well the theoretically expected results (Fig. 5).

In the second set of experiments, a larger N52 neodymium cylindrical disk magnet was used with a diameter of $50 \mathrm{~mm}$, a thickness of $10 \mathrm{~mm}$ and a commercial rating of $45 \mathrm{~kg}$ pullforce. The target specimen shapes and sizes were varied to see how they affect the magnetic force curve as well as a neodymium magnet as a target specimen was also introduced to observe how significantly greater strength it provides. The results of Experiment 1 combined with the results of Experiment 2 demonstrated the effect of the size and the material of the target specimen. The choice of the type of ferromagnetic steel (mild steel, silver steel) did not show a notable difference. The size and shape of the target specimen resulted in a slightly more notable difference where it was found that the force does not necessarily increase linearly with the increase of volume, but that the area perpendicular to the magnetic field lines is of more importance (Fig. 6). This was particularly seen between the short and long rod magnet where the former had a larger volume but smaller area perpendicular to the magnetic field lines and thus performed slightly worse than the long rod magnet with smaller volume but larger area perpendicular to the magnetic field lines. Even though changes in such shapes of steel specimen was found to make a difference in the force results, the change of force values between choosing a target specimen made from steel versus from neodymium magnet made an order of magnitude larger difference, as shown from the results of Experiment 2. 

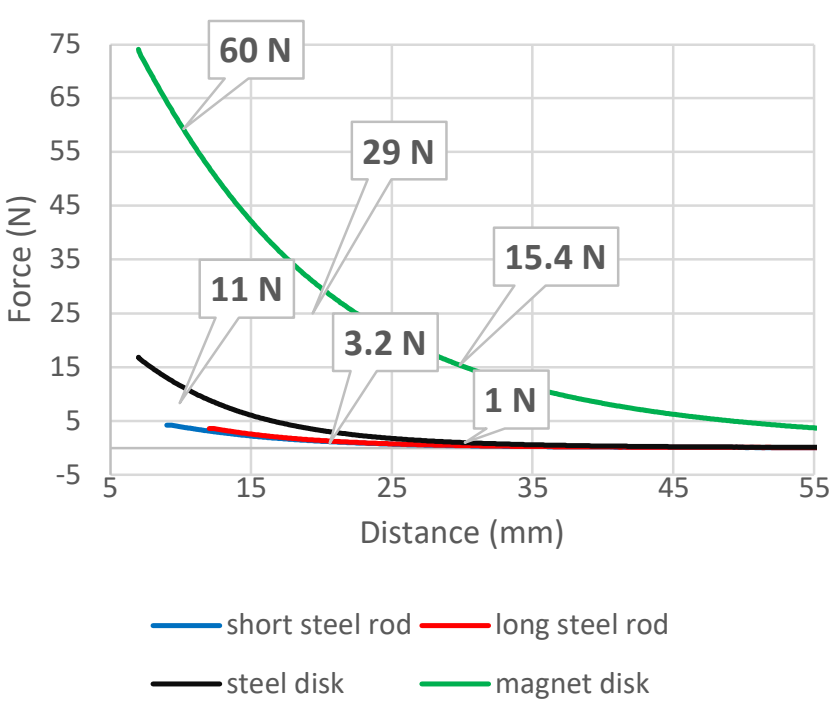

Figure 6 - Force curves produced by varying the distance between a $45 \mathrm{~kb}$ pull-force neodymium magnet and a target specimen of a short steel rod, a long steel rod, a steel disk and a disk magnet .

Therefore, it can be concluded that it is much more efficient to use magnetic material for the frame of the deployable structure.

In Experiment 3, the effect of the medium between the magnet and the target specimen was investigated. During the application of the magnetic technology, soft tissues such as the abdominal wall, skin and muscle tissue would be in-between the external magnet and the target structure. For this reason, a soft tissue sample was placed between the magnet and the target specimen to observe the effect it may make on the magnetic field. For the soft tissue phantom, a bag of diced chicken liver was used with a thickness of around $20 \mathrm{~mm}$. The experimental setup was the same as in Experiment 2 where the target specimen was chosen to be the $25 \mathrm{~kg}$ pull-force neodymium disk magnet and the bag of diced liver was placed between the two magnets.
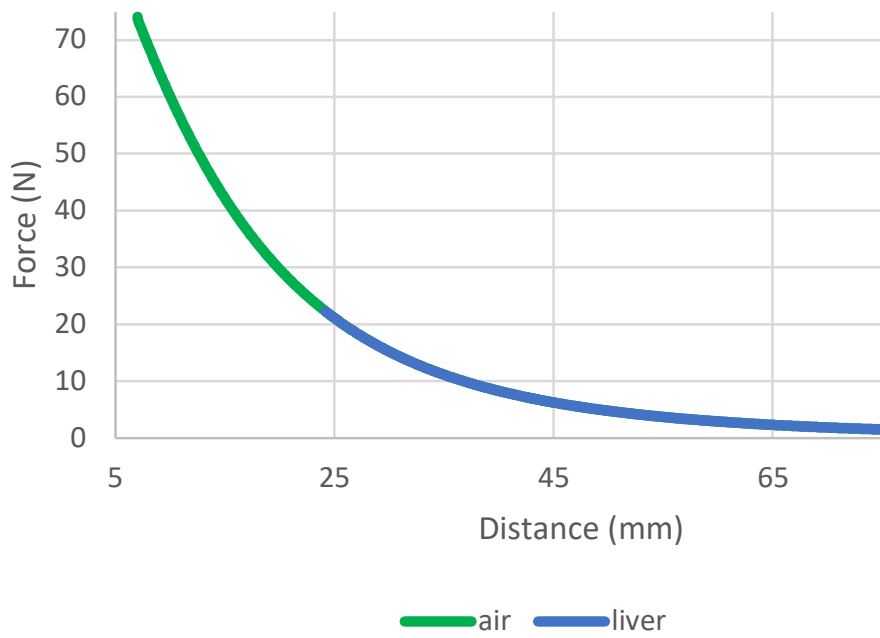

Figure 7 -Force curve between a $45 \mathrm{~kg}$ pull-force neodymium magnet and a $25 \mathrm{~kg}$ pull-force neodymium magnet as the target specimen with and without placing a $20 \mathrm{~mm}$ thick bag of diced chicken liver in-between.
As expected, Experiment 3 showed that nonferromagnetic tissue has virtually no influence on the magnetic field, therefore the amount of soft tissue such as the abdominal wall including the skin, muscle and fat would not notably weaken the magnetic attraction (Fig. 7).

In Experiment 4, one of the strongest diametrically magnetized N52 neodymium rod magnet was chosen as the target specimen that would still comfortably fit in a $10 \mathrm{~mm}$ standard trocar. This specimen was tested against one of the strongest grade N52 neodymium disk magnet of $50 \mathrm{~mm}$ diameter and $10 \mathrm{~mm}$ thickness. The experiment was set up identically to Experiment 2 . When positioning the diametrically magnetized rod magnet it was crucial to position it in such a way that its opposite pole faced the $45 \mathrm{~kg}$ pullforce disk magnet. This was achieved by placing an acrylic bar between the two magnets to allow for opposite magnetic pole self-alignment after which the poles were marked on the rod magnet with a permanent marker. This experiment gave an insight in the order of magnitude of achievable magnetic forces at various distances under such conditions. At $20 \mathrm{~mm}$, the considered abdominal thickness, the forces produced in

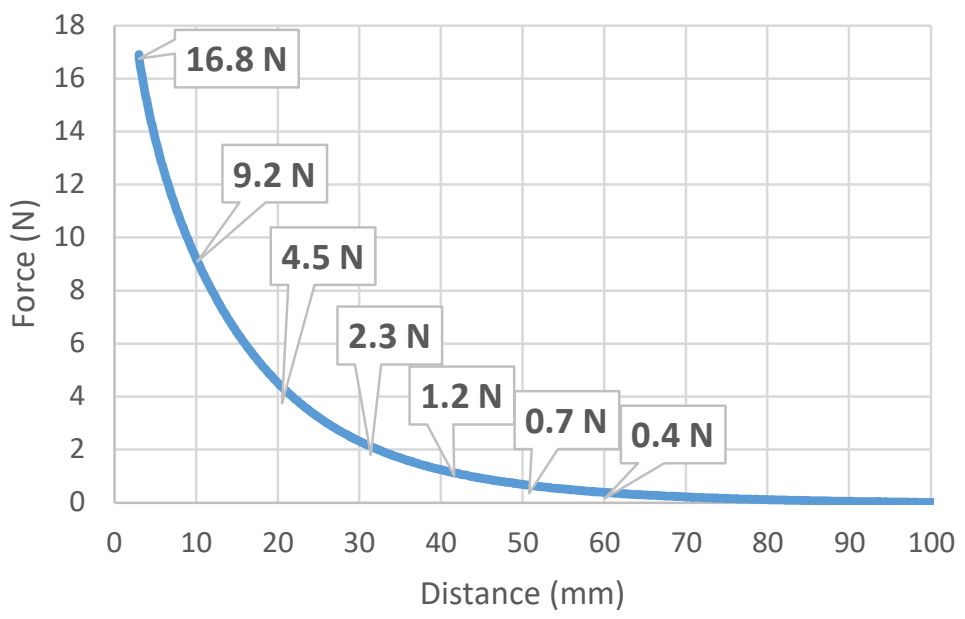

Figure 8 - Force curve produced by a $45 \mathrm{~kg}$ pull-force disk magnet and a $6.9 \mathrm{~kg}$ pull-force diametrically magnetized rod magnet.

Experiment 4 are 4.5 N. If 3 of these magnet pairs are used, that would provide a total of $13.5 \mathrm{~N}$ lifting force, which already sufficiently meets the force requirements of holding the liver with $11.25 \mathrm{~N}$ force or greater (Fig. 8). Therefore, these experiments laid an evidence-based foundation to the feasibility of magnetic technology to anchor the liver during surgery.

\section{DISCUSSION}

As part of our ongoing research, an innovative liver retraction solution was proposed with non-invasive magnetic anchoring that could be used primarily in manual laparoscopic procedures. The proposed solution has the potential of making a significant clinical impact once the device is built, tested and having passed clinical and regulatory approval requirements. It could change the way upper abdominal procedures are done where the liver retraction still poses a significant technical and surgical challenge [12]. 
Minimizing tissue damage and increasing the visibility and exposure of the Calot's triangle or the hiatus were the primary focus of attention whilst developing the proposed liver retracting solution. Proposing magnetic anchoring could completely eliminate the need of any additional incision on the abdominal wall, thus significantly decreasing the chance of post-operative hernia development [13]. Internal tissue damage is also reduced by not grasping or puncturing the liver for retraction. The three bands would create a larger surface area when compared to the Versa Lifter Band [11] that only uses one band or other retraction solutions that use strings [14] for retracting the liver. The only internal tissue damage is caused on the right crus of the diaphragm if the first proposed string/band solution is applied. This minimal tissue damage could even be further decreased if the accompanying string/band solution used a different technique to draw the liver to the internal anchoring structure for instance by looping around the liver without the need of grasping the diaphragm. The proposed solution with three bands internally anchored would sufficiently fold either lobe of the liver away to provide a large enough workspace visibility.

On a larger scale, the magnetic anchoring solution could be applied to a range of other procedures with adjusted accompanying features for practically any other tissue retraction. For example, the use of such a device could also be extended to typical lower abdominal procedures such as for prostatectomy and hysterectomy procedures where tissue retraction such as of the bladder is also a crucial need; or for any procedures on the lower gastrointestinal tract where retraction of the small intestines still poses a challenge [15].

Lastly, the technology could also have a significant scientific impact as an initial proof of concept that could encourage other researchers to further investigate the potential of magnetic tissue retraction in MIS procedures that could lead to the development of a new specialized field of medical device development. Arguably, the future of retraction is robotic, numerous research experiments already suggested ways to execute this task autonomously, or with robotic support, yet, even in those future cases, advances retractor designs will be needed $[4,7,16]$.

\section{CONCLUSION}

Our research aims to create a detailed medical device design, a magnetic anchoring system suitable for liver retraction, wherein the safety and efficacy of the method was to be investigated at first. The proposed technology was critically analyzed using experimental laboratory work that provided sufficient evidence proving the potential of the concept and its intended use and application. The experimental work investigated the feasibility of the magnetic attachment, primarily quantifying the effect of the varying distance between the external magnets on the abdominal wall surface and the internal magnetic anchoring structure. The experimental results showed that with commercially available external disk magnets coupled with three internal rod magnets $13.5 \mathrm{~N}$ perpendicular magnetic forces can be achieved, which is sufficient to hold the weight of an average liver, requiring $11.25 \mathrm{~N}$ forces. The detailed design included considerations of the anatomical features of the patient's abdominal wall and how the device would consist of joints allowing the required freedom of movement without jeopardizing structural integrity, device- and patient-safety. The solution design showed a potential for a significant scientific and clinical impact where the device would decrease tissue damage, recovery time, technical challenge and improve both patient outcomes, surgical staff experience and work efficiency.

\section{ACKNOWLEDGMENT}

T. Haidegger's work is partially supported by the ELKH SZTAKI - OE Cyber-Medical System Development project. T. Haidegger is supported through the New National Excellence Program of the Ministry of Human Capacities. He is a Bolyai Fellow of the Hungarian Academy of Sciences.

\section{REFERENCES}

[1] Hoeckelmann, Mathias, Imre J. Rudas, Paolo Fiorini, Frank Kirchner, and Tamas Haidegger. "Current capabilities and development potential in surgical robotics." International Journal of Advanced Robotic Systems 12, no. 5 (2015): 61.

[2] Haidegger, T., 2019. Probabilistic method to improve the accuracy of computer-integrated surgical systems. ACTA POLYTECHNICA HUNGARICA: JOURNAL OF APPLIED SCIENCES, 16(8), pp.119140 .

[3] Nagy, T.D. and Haidegger, T., 2019. A dvrk-based framework for surgical subtask automation. Acta Polytechnica Hungarica, 16(8), pp. 61-78.

[4] Haidegger, Tamás. "Autonomy for surgical robots: Concepts and paradigms." IEEE Transactions on Medical Robotics and Bionics 1, no. 2 (2019): 65-76.

[5] O’Shea, Conor, Denis Kelliher, Emmet Andrews, Mícheál O’Ríordáin, Michael O'Shea, Timothy Power, and Pádraig Cantillon-Murphy. "Design, development and evaluation of an inflatable retractor for atraumatic retraction in laparoscopic colectomy." Annals of biomedical engineering 42, no. 9 (2014): 1942-1951.

[6] Takács, Árpád, Imre J. Rudas, and Tamás Haidegger. "Surface deformation and reaction force estimation of liver tissue based on a novel nonlinear mass-spring-damper viscoelastic model." Medical \& biological engineering \& computing 54, no. 10 (2016): 1553-1562.

[7] Nagy, T. D., M. Takács, I. J. Rudas, and T. Haidegger. "Surgical subtask automation-Soft tissue retraction." In 2018 IEEE 16th World Symposium on Applied Machine Intelligence and Informatics (SAMI), pp. 55-60. IEEE, 2018.

[8] P. Chiu, B. Leung, S. Sy, C. Chan, S. Wong, C. Poon, "The safety and efficacy of a new liver retractor for the performance of laparoscopic upper gastrointestinal surgery". 2013 Scientific Session of the Society of American Gastrointestinaland Endoscopic Surgeons (SAGES), P386

[9] Shah, Dignesh, Andrew Alderson, James Corden, Thomas Satyadas, and Titus Augustine. "In vivo measurement of surface pressures and retraction distances applied on abdominal organs during surgery." Surgical innovation 25, no. 1 (2018): 50-56.

[10] Sucandy, Iswanto, Geoffrey Nadzam, Andrew J. Duffy, and Kurt E. Roberts. "Two-port laparoscopic cholecystectomy: 18 patients human experience using the dynamic laparoscopic NovaTract retractor." Journal of Laparoendoscopic \& Advanced Surgical Techniques 26, no. 8 (2016): 625-629.

[11] Genser, Laurent, Adriana Torcivia, Arnaud Saget, Jean-Christophe Vaillant, and Jean-Michel Siksik. "Minimal invasive internal liver retractor in conventional and trans-umbilical single-incision laparoscopic sleeve gastrectomy: video report." Obesity surgery 26, no. 3 (2016): 704-705.

[12] Cavallo, Aida, Margherita Brancadoro, Selene Tognarelli, and Arianna Menciassi. "A soft retraction system for surgery based on ferromagnetic materials and granular jamming." Soft robotics 6 , no. 2 (2019): 161-173.

[13] Sajid, Muhammad S., Syed A. Bokhari, Ali S. Mallick, Elizabeth Cheek, and Mirza K. Baig. "Laparoscopic versus open repair of incisional/ventral hernia: a meta-analysis." The American journal of surgery 197, no. 1 (2009): 64-72.

[14] Palanivelu, Praveenraj, Kedar Pratap Patil, Ramakrishnan Parthasarathi, Jaiganesh K. Viswambharan, Palanisami Senthilnathan, and Chinnusamy Palanivelu. "Review of various liver retraction 
techniques in single incision laparoscopic surgery for the exposure of hiatus." Journal of minimal access surgery 11, no. 3 (2015): 198.

[15] O’Shea, Conor, Denis Kelliher, Emmet Andrews, Mícheál O’Ríordáin, Michael O'Shea, Timothy Power, and Pádraig Cantillon-Murphy. "Design, development and evaluation of an inflatable retractor for atraumatic retraction in laparoscopic colectomy." Annals of biomedical engineering 42, no. 9 (2014): 1942-1951.
[16] Attanasio, Aleks, Bruno Scaglioni, Matteo Leonetti, Alejandro F. Frangi, William Cross, Chandra Shekhar Biyani, and Pietro Valdastri. "Autonomous Tissue Retraction in Robotic Assisted Minimally Invasive Surgery-A Feasibility Study." IEEE Robotics and Automation Letters 5, no. 4 (2020): 6528-6535. 